\title{
Research on the Status Quo and System Architecture of the Web Information Resource Evaluation
}

\author{
Wei Xu, Ji Liu, and Xiangxing Shen \\ School Information Management, Wuhan University, Wuhan 430072, \\ China \\ wilson_hu@126.com, sim@whu.edu.cn, x.shen@whu.edu.cn
}

\begin{abstract}
Web information resource evaluation (website evaluation) becomes more and more important, as the web information resources become larger and more complex. Thus, a lot of theory and methods have been developed to evaluate websites. In this paper, the author analyzes the primary methods applied currently in the evaluation of the web information resource and points out the main problems in this area from the perspectives of qualitative evaluation, quantitative evaluation, synthetic evaluation and automatic evaluation, after making an in-depth and comprehensive research of the literature and evaluation tools at home and abroad. Furthermore, the paper concludes that the evaluation of information resource on the Internet should take the internal and external characteristics into consideration, in the meantime, the paper puts forward a system architecture scheme of the automatic website evaluation based on the usability engineering.
\end{abstract}

\section{Introduction}

Internet, since its birth several decades ago, has published far more information than any traditional media. According to the 19th Survey Report [1] published in January, 2007, until December 31st, 2006, the number of computers accessed to the Internet in China mounts to $59,400,000$, which is increased by $20.0 \%$ by comparing to the number last year; websites are altogether 843,000 , increased by $21.4 \%$.

The rapid development of the Internet on one hand stimulates the dramatic growth and accumulation of information. On the other hand, it brings about a large

Please use the following format when citing this chapter:

Xu, W., I.iu, J., Shen, X., 2007, in IlIP International lederation for Information Processing, Volume 252, Integration and Innovation Orient to L-Society Volume 2, eds. Wang, W., (Boston: Springer), pp. 529-539. 
amount of junk information and jam information, which results in serious information overload. A great many researches show searching for information is the main purpose for individuals to surf on line. However, in the searching period, about $60 \%$ of the time is wasted on futile work. For organizations, about $50 \%$ prospective users are lost because they cannot find the information they need. The common demand of individuals and organizations makes the digital information resource management [2], efficient search and evaluation imperative under the situation. Web information resource (websites), as the principal information carrier in cyberspace, plays a key role as the information node in the network. Therefore, the evaluation and method selection become more important than ever in the age of everaccelerating information. Presently, foreign countries have set about the research on website evaluation; China also launches the projects too. But the large numbers of evaluation methods do not mean this area has become mature. The underlying reasons are due to the complexity of the websites themselves. The difference of website platform, implementing technology, positioning, contents and dimensions cause enormous obstacles for website analysis in reality. The large number of evaluation methods reveal the scarcity of the consistent theoretical guide and precise implementing frame. Confronting with the complicated network environment, without the uniform theoretical guide or scientific methodology, it will be difficult to collect and associate the separated problems of details, find out the crux and direction for improvement and ensure the accuracy and reliability of the analysis and evaluation results.

\section{Researches on the Status Quo of Website Evaluation}

\subsection{Qualitative Evaluation}

Qualitative evaluation mainly contains two methods: indexes system and questionnaire. Indexes system is to detect the quality of the website through setting a set of indexes. The indexes will vary according to the purpose, perspective and type of websites. An oversea scholar Betsy Richaman put forward 10C [3] principles; Harris Robert established four standards for website evaluation [4]; David Stoker and Alision Cooke raised 8 criteria [5] which are totally different from Harris's; Gemer L. Wilkinson, etc. compared and summarized the evaluation criteria brought by scholars in the thesis Index Listing of Evaluation Criteria and Quality. Based on the comprehensive analysis of the characteristics and attributes of websites, they concluded 125 qualitative website evaluation indexes in 11 categories [6]. Domestic scholars provided relevant index systems for websites of different types, for instance, the library website evaluation index system [7] and literature [8] discuss the synthetic evaluation index system from the angles of website design, contents and technology. Questionnaire makes best use of the users' recognition, perception and attitude to the websites to measure the impact they work on the users. This method is often carried out by evaluation organization or individual to know the serving 
capacity and operating effect of the website. They make a series of questionnaire, conducting the research, analyze the collected data and evaluate the website quality.

Qualitative evaluation can analyze the website quality roundly, make full use of the individual's perception of the website, and acquire direct and understandable results. But it still has following problems.

\subsubsection{Some Problems Exit in Indexes System Method}

- The index system doesn't cover all the main attributes. Website is a very complicated and enormous system. The evaluation indexes are complex too. Until now, the differences of index systems raised by scholars and evaluation institutes and the disagreement on this issue display this point clearly. As a matter of fact, few have not conducted comprehensive and synthetic observation of websites, nor have they engaged in objective unveiling of the internal and external attributes of the websites.

- Some of the indexes are not reasonable. The websites have many attributes. There are cause-and-effect relations between each of them. Some of the evaluation methods have not given scientific positioning for the indexes, for example, the relation between the quality of the information, website traffic, page views and connectivity is usually defined separately. Repetitions often occur in indexes, for example, although there are some differences in the expression of indexes, the essence meaning is quite the same or similar (e.g. credibility and accuracy). Some indexes are hard to understand, such as critical thinking. Some indexes seem reasonable, but the practical examination and application are really hard. They are not feasible. For example, the indexes such as justice and perception of the world can not be taken as the criteria to evaluate the website quality.

- Some indexes are aimed at ambiguous objects. The website is constituted by the framework as well as the information content. The evaluation indexes of webpage structure and information content are not properly distinguished. Some indexes are applicable for webpage evaluation, while some are applicable for information content. Blurring the boundary between them confuses the objects and makes them not scientific.

\subsubsection{Some Problems Exit in Questionnaire Method}

During the process of implementing questionnaire, the error is difficult to control, especially the validity and objectivity of the users' answers, which affects the accuracy of the quality research results.

Besides, due to the strong subjectivity of qualitative evaluation, the evaluation result is easy to be impact by the network environment, evaluation standard, as well as the qualification and emotion of the evaluation staff. This causes this method not so feasible. The reliability of the results is weakened too. Time, human endeavor, facility and money will be increased in this case. The cost is too high. It can hardly meet the demand of massive websites evaluation. 


\subsection{Quantitative Evaluation}

Quantitative evaluation provides a series of scientific, normative and objective evaluation methods, illustrating and solving the problem by reliable figures. Originally, it evolved from the analysis of the basic elements such as connectivity, page views and so on, developed into a systematical quantitative evaluation method. The three typical quantitative evaluation methods applied abroad include Webometrics (Link Analysis), analytical hierarchy process and correspondence analysis.

\subsubsection{Webometrics}

Webometrics, adopting traditional citation analysis theories, is an efficient method applied in website evaluation. It is taken for granted that the more linkage a website has, the higher the quality is. Research shows the research of the relation between the webpage links is somewhat similar to that of the citation research of the published articles. But they still have some nuance. This is in accordance with Lotka effect [9]. When the linkage analysis is applied in research, some famous searching engines such as Google and Alta Vista are used. These engines can usually offer some special software interface and data searches method, which helps the users to find out the data they need.

\subsubsection{Analytical Hierarchy}

Analytical hierarchy process utilizes human's conventional thoughts to solve problems. It divides a complicated problem into several detailed problems, making full use of the analysis, judgment and integration capacities, and quantifies the complexity. This method makes the conception classification of website attributes, collecting and measuring the data by the experts' investigation. In this way, it evaluates the grades and ranks of websites, such as some literature [10] explores into the trains of thought and method of the application of analytic hierarchy process to university libraries websites evaluation.

\subsubsection{Correspondence Analysis}

Correspondence analysis (Relational Analysis) was originally raised by a French scientist and adopted in enterprise decision-making positioning analysis. The English professor Berthon applied correspondence analysis to evaluate 15 websites of the telecommunication company in the world in 2001 [11]. It was proved to be a breakthrough in this area. He pointed out correspondence analysis could solve the positioning problems of the website evaluation; distinguish the exact difference between the websites. This method can not only be helpful in telecommunication websites evaluation, but also websites of different kinds. The literature [12] used correspondence analysis to make an experimental evaluation of the media website in China. It established a mathematical model of this analysis through defining the basic attributes of the websites, collecting relevant data from Alexa [13] database and generating the correspondence positioning graph. This can be involved in decision-making positioning analysis and has great reference value.

Quantitative evaluation to some extent overcomes the problems of subjectivity and controllability in qualitative analysis. Whereas, it still has many problems too. 
- The limitation of link analysis

As the research goes further, it is discovered that there are spectacular differences between literature citations and website connections. Many problems arise. For example, Stephen P. Harter and Charlotte E. Ford discover in their research, among the links to the e-print websites, nearly half of them are linked to themselves, which is helpless for the evaluation of literature academic value and research on academic communication; among the webpage linked to e-periodical websites, the percentage of the academic websites is lower than $8 \%$. Thelwall [14] analyses the 100 webpage linked with the highest frequency by the English universities. The result also shows the linkage can not indicate the academic value of the websites. Currently the reached agreement is that the links of the webpage is substantially different from the citation. Although citation analysis can illuminate the linage research, considering the difference between them, the results concluded from it should be processed accordingly.

- The limitation of analytical hierarchy process

There are two limitations of the application of analytical hierarchy process to website evaluation. First, the process itself has some disadvantages. Secondly, the process still has some problems in adjusting with the website evaluation. The classification and measurement of analytical hierarchy process are carried out by human beings. So is the scoring procedure. The error control is enforced, but cannot avoid human subjectivity, which causes some deviation between the result and reality. The component elements of the websites are also interacted. Even if the relationships between the elements, the general and simple classification of the hierarchy worked out by the process still makes the results deviate the reality.

- The limitation of correspondence analysis

Since correspondence analysis only evaluates the information of website positioning and distinctions, it is not based on the integrity of the website. Therefore, it can hardly reflect the synthetic level of the website roundly and systematically. On the other hand, the data of the data warehouse also have defects. They can be modified easily and may have some errors in data check, which causes obstacles to the practical work of website evaluation.

Up to date, quantitative evaluation of websites adopts traditional theories and methods which provide a useful train of thought and consulting reference in website evaluation but result in the neglect of the characteristics of the websites themselves and further research aimed at the users. The scarcity of a scientific positioning of evaluation objects is also one of the disadvantages. It has not made any substantial development in evaluation, but still has the consulting value in academic research.

\subsection{Synthetic Evaluation}

Synthetic evaluation includes the synthetic method based on user research and quantity detecting, website evaluation based on Information Architecture (IA) theory and website framework evaluation. 


\subsubsection{Website Evaluation Based on IA Theory}

This method conducts access analysis [15] from four systems: the classification, navigation, searching and labeling of the content of the IA. It is usually carried out from the angle of the user experience, evaluating the website by making the users define the application target and then checking their performance in practicing. By comparing with the qualitative evaluation, it is more systematical and scientific, belonging to the demonstrating analysis category.

\subsubsection{Website Framework Evaluation}

This method accords to the different profiles of the website, establishes a set of measuring standard, putting all the standard indexes in the structure frame and table, regarding the indexes as the criteria of website evaluation. This method can be described objectively and quantified by the quantitative criteria. The evaluation result is comprehensive. It is a compromise.

Since synthetic evaluation applies the merits of both qualitative and quantitative evaluation, it can obtain better results. But two questions should be coped with properly: how to decide the proportion of qualitative and quantitative evaluation. If it is taken randomly, error will occur in the evaluation system. While actually the efforts and costs spent on this method are far more than merely using qualitative or quantitative method.

\subsection{Automatic Evaluation}

Automatic evaluation sets about from the attributes and characteristics of the websites, utilizes digital, automatic and intelligentzed means to solve the problems. It develops relevant automatic detecting software or websites, makes them available for all websites testing and evaluation data automatic collecting, according to the established website evaluation model, conducts the Stat, analysis and calculation automatically, and then works out the evaluation result. The automatic testing takes counter, Cookies, cache memory and IP address as the basic analytic data, on the ground of the user's page view record analysis, applying the analysis on the Web sites and automatic analysis on the server log, etc. to evaluate the load and quality of the information on the website objectively. It is objective and credible [16].

Though automatic evaluation can detect some more relational mistakes, enhance the coverage of the analyzing features, reduce the experts' dissatisfaction and time consumption and fuse with usability interface (UI) design and analysis. But automatic analysis can only be taken as the standard analysis. Different techniques reveal raise different problems. The subjective evaluation on the user's satisfaction can not adopt automatic testing. Meanwhile, this area is still premature. The reason for the phenomenon lies in the complexity of the website itself. Users want to know and observe the data. But it is too complicated to be practicable. In this case, it can merely be regarded as the reference in website evaluation. 


\section{Architecture of Website Evaluation}

\subsection{Setting of the Evaluation Index System}

Website evaluation must be implemented based on its own characteristics, under the guidance of the theories of this area. The existent research and practice have not distinguished the attributes of the websites properly, leading to the chaos of the setting of index system. The author believes, for a website, it has internal and external characteristics, reflecting its quality from different angles. Thus the evaluation should be carried out from these two standpoints.

\subsubsection{Website Internal Characteristic}

The internal characteristic of the website is decided by the component elements of the website entity. It mirrors the technological implementation of the website target. Newman and Landay's research [17] shows website is a multidimensional compound which covers content, navigation and appearance [18], just as what Figure 1 indicates.

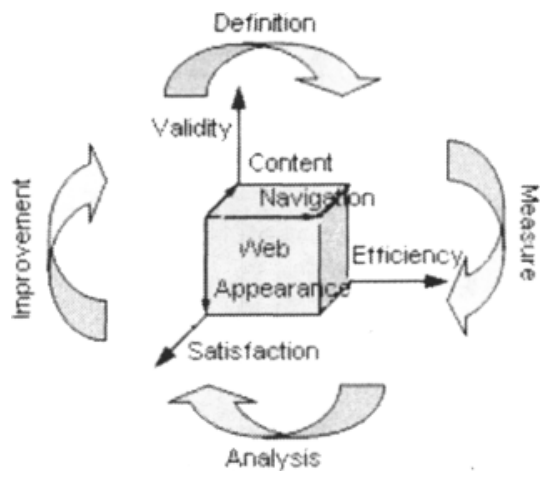

Fig. 1. Website internal evaluation objects

This distinction can avoid the interaction of the elements. Hence analytic hierarchy process may help to set scientific indexes. Content evaluation focuses on the information construction. In detail, it includes four aspects [19]: sorting system, search system, index system and information security system. Navigation evaluation needs to involve global navigation, local navigation, supplementary navigation and semantic navigation evaluation. The appearance evaluation judges the display and distribution, for example, the color collocation of the website, selection of multimedia materials and so on. This process researches the objective attributes such as content, navigation and appearance thoroughly. By applying the hierarchy 
analysis, the weight will be ascertained; the website internal characteristic evaluation index system will be established.

\subsubsection{Website External Characteristic}

Website external characteristic includes Web traffic, visit, Connectivity, Speed, Page Views, user's average page views and freshness as well as the number of the registered users and their website application frequency. The ultimate target is to serve the user. The users' degree of satisfaction and indexes related to the user experience are the core of the external characteristic. As it is showed in Figure 2, acquiring the data, adopting correspondence analysis to conduct cluster analysis and elaborating the distinctions of the websites in the network are a helpful supplementary factor in website evaluation [20].

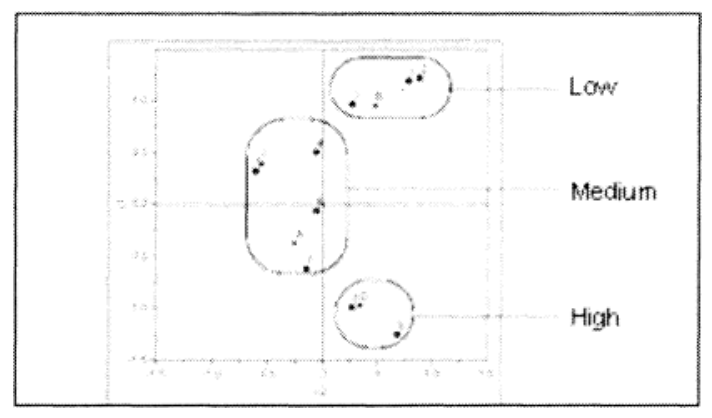

Fig. 2. Cluster analysis graph of correspondence analysis

The problems, such as ambiguous positioning still exist in the previous index system, index unable to reflect the website quality, defects and limits are found in the adaptability of analytic hierarchy process and correspondence analysis, can be revealed through the characteristic analysis of the websites. This will be helpful to form a series of indexes which are mainly supported by qualitative evaluation and assisted by quantitative evaluation.

\subsection{Architecture of Automatic Evaluation System}

The system adopts software fast prototype method, combining with the ObjectOriented Design methods. It is used to develop automatic website evaluation tool model system based on $\mathrm{B} / \mathrm{S}$ model. The development process will involve Java or NET technology, UML, XML, Web Services, Intelligent Agent and Portal techniques, applying the multi-layer software structure grounded on J2EE and NET Framework. Maybe it can realize the expansibility and maintainability of the software platform as Figure 3 shows. 


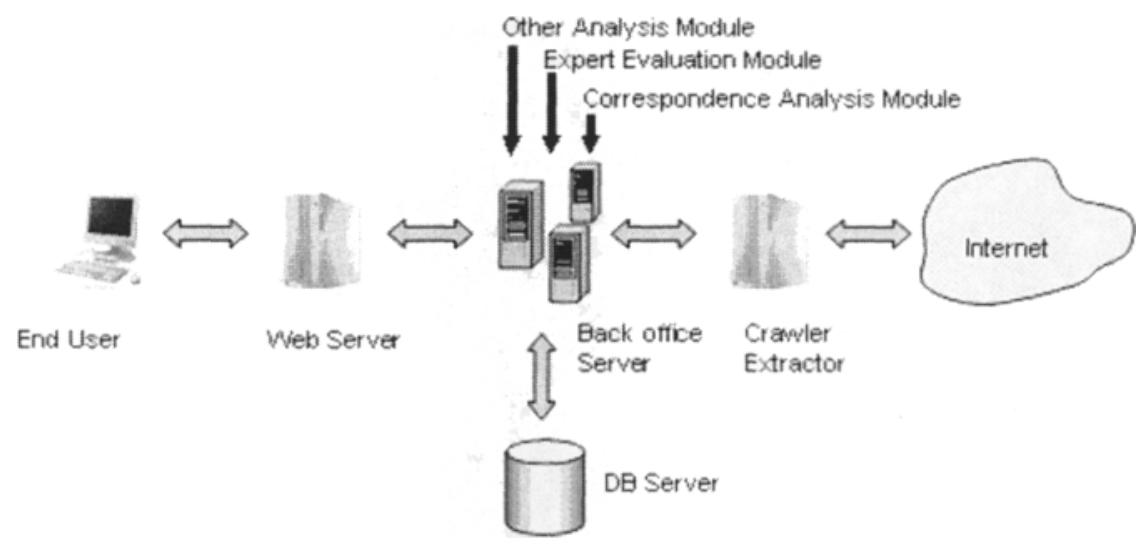

Fig. 3. Structure of the automatic website evaluation system

\subsubsection{End User}

Terminal user uses personal computer to visit all the Web Serves to get the evaluation (main information) of different websites and basic website development information (supplementary information).

\subsubsection{Web Server}

Web server provides Web browse service, applying program interface and component interface through distribution scheme to use the background server application module.

\subsubsection{Back Office Server}

Back office server is the critical and difficult part of the system development. The development is implemented on the basis of the development of the open software system of J2EE or NET Framework, etc to realize the dynamic increase and adjustment of the automatic website analysis tools. The main components include Profile developing tool, matrix calculating tool and analysis calculating tool, etc. The key is to establish a standard website characteristics corpus and an intelligent analytic comparing algorithm of the development. The website parameter standard characteristic corpus, such as the website information construction standard, color standard and navigation standard, etc., should vary according to the types of different websites in order to offer the reference to comparative analysis. The parameter standard corpus should include the weights of parameters raised by the experts. They should vary with the types of different websites too. Intelligent analysis tools can be functionally divided to three systems: relational analysis, comparative analysis and indirect measurement. Relational analysis system is applicable in website positioning and distinguishing to expel relevant interference factors. Comparative analysis system is used to compare the eigenvalues of the parameter standard corpus and websites [21]. It combines with the calculation and corresponding analytic matrix, 
and conducts an all-round analysis of all the components inside the website. Indirect measurement system is adapted to measure two comparatively subjective indexes of the user's degree of satisfaction and website practice experience. Meanwhile, it indirectly indicates the analysis of the characteristic parameters of the two indexes in order to evaluate the user's satisfaction degree and practice experience [22].

\subsubsection{Crawler and Extractor}

Crawler and extractor is the crucial component in collecting the objective website data [23]. It utilizes the well-shaped searching engines and the characteristics of the system itself, develops the extractor which is suitable for the system. Accords to the set index system, it provides objective data to the automatic website evaluation. The extractor observes the established requirement; captures the internal and external characteristics data of the website, converts them into needed forms and extracts the objective data of the demanding websites.

\subsubsection{DB (Data-Base) Server}

The data in the system is stored in the database. It includes the data of the benchmark website, data of the target website evaluation, matrix generated or analyzed in calculation and data of the users' page views. In practice, the system will apply distributed database to balance the load.

\section{Conclusions}

At present, applied website evaluation is rare. The methods raised until now cannot evaluate all the indexes of a website roundly. Therefore, on the base of a systematical conclusion and research of the achievements in the area home and abroad, it is necessary to explore the automatic website evaluation model and method grounded on usability engineering theories. It will be useful for the perfect of information resources web site evaluation indexes system. evaluation model and evaluation mechanism, optimizing the information web site configure, promoting the government function conversion for fulfilling the market supervision function and common management function.

\section{Acknowledgment}

This paper is the Major Project of Philosophical and Social Science of Ministry of Education, Management and Utility of Digital Information Resources (05JID00024).

\section{References}

1. China Internet Network Information Center, The 19th survey report (February 18, 2007); http://www.cnnic.net.cn/en//index/0O/02/index.htm.

2. W.C. Wu and J.Z. Zhang, "Study of network information standardization system", Information Science. 19(1), 40-46 (2001).

3. J. Su, "Research on the network information resources evaluation", Journal of Academic Libraries. 23(1), 7-13 (2005). 
4. W.J. Chen and Y.S. Chen, "Commentary on evaluation of network information resources", Journal of the Library Science Society of Sichuan. 40(1), 25-3I (2004).

5. J. Shen and Q.H. Zhu, "Current research on evaluation criteria of networked information resources in China and overseas", Information Science. 23(7), 1104-1109 (2005).

6. J.L. Zhao and L.J. Chen, "Research summary on the network information resources evaluation overseas", Library Work and study. 18(3), 24-26 (2005).

7. H.T. Zhang, "A study of indicators for the evaluation of library website", Document Information \& Knowledge. 25(2), 96-99 (2005).

8. D.M. Li, "Preliminary exploration of website integrative evaluation", Information studies Theory \& Application. 28(2), 303-306 (2005).

9. R. Rousseau, "Sitations: An exploratory study", Cybernetics. 1(1), 1-9 (1997).

10. H.M. Wei, "Application of analytical hierarchy process in libraries webs evaluation", New Technology Library and Information Service. 29(10), 74-83 (2005).

11. P. Berthon, "Positioning in Cyberspace: Evaluating Telecom Websites Using Correspondence Analysis", Information Resources Management Journal. 14(1), 13-21 (2001).

12. X.S. Shen and D.M. Li, "Research on China network media websites evaluation: Using correspondence analysis", China Soft Science. 19(1), 126-133 (2005).

13. "Alexa Internet Inc, Alexa Web Search Platform" (December 13, 2006); http://www.alexa.com/.

14. M. Thelwall, "An initial exploration of the link relationship between UK university Web sites", Aslib Proceeding. 52(2), 118-126 (2002).

15. L. Gan, "Examination \& analysis of information architecture of e-commerce website", Information Studies Theory \& Application. 28(6), 605-608 (2005).

16. D.M. Li, "The research on the automated website information evaluation", Document Information \& Knowledge. 25(1), 104-107 (2005).

17. M.W. Newman, and J.A. Landay, "Sitemaps, storyboards, and specifications: A sketch of web site design practice", Proceedings of Designing Interactive Systems (New York, 2000), pp. 263-274.

18. J.X. Hao and X.X. Shen, "Study on the framework for website analysis based on usability engineering", Document Information \& Knowledge. 26(2), 81-86 (2006).

19. Y.H. Rong and Z.P. Liang, "An approach to information architecture", Journal of the China Society for Scientific and Technical Information. 21(2), 229-232 (2003).

20. X.X. Shen, et al, "Evaluating China's university library websites using correspondence analysis", Journal of the American Society for Information Science and Technology. 57(4), 493-500 (2006)

21. Chi, Ed H, et al, "The bloodhound project: Automating Discovery of Web Usability Issues Using the Info Scent TM Simulator", Proceedings of the Conference on Human Factors in Computing Systems (ACM Press, New York, 2003), pp. 505-512.

22. B.J. Cockrell, Jayne, and E. Ahow, "Do I Find an Article? Insights from a Web Usability Study", The Journal of Academic Librarianship. 28(2), 54-64 (2002).

23. J.I. Hong, et al, "Web Quilt: A proxy-based approach to remote web usability testing", ACM Transactions on Information Systems. (19), 263-285 (2001). 